\title{
Incidence and Trends of Infections with Pathogens Transmitted Commonly Through Food and the Effect of Increasing Use of Culture-Independent Diagnostic Tests on Surveillance — Foodborne Diseases Active Surveillance Network, 10 U.S. Sites, 2013-2016
}

\begin{abstract}
Ellyn P. Marder, MPH${ }^{1}$; Paul R. Cieslak, MD²; Alicia B. Cronquist, $\mathrm{MPH}^{3}$; John Dunn, DVM ${ }^{4}$; Sarah Lathrop, PhD ${ }^{5}$; Therese Rabatsky-Ehr, MPH' Patricia Ryan, $\mathrm{MD}^{7}$; Kirk Smith, DVM ${ }^{8}$; Melissa Tobin-D’Angelo, MD ${ }^{9}$; Duc J. Vugia, MD ${ }^{10}$; Shelley Zansky, PhD ${ }^{11}$; Kristin G. Holt, DVM ${ }^{12}$; Beverly J. Wolpert, $\mathrm{PhD}^{13}$; Michael Lynch, $\mathrm{MD}^{1}$; Robert Tauxe, $\mathrm{MD}^{1}$; Aimee L. Geissler, $\mathrm{PhD}^{1}$
\end{abstract}

Foodborne diseases represent a substantial public health concern in the United States. CDC's Foodborne Diseases Active Surveillance Network (FoodNet) monitors cases reported from 10 U.S. sites* of laboratory-diagnosed infections caused by nine enteric pathogens commonly transmitted through food. This report describes preliminary surveillance data for 2016 on the nine pathogens and changes in incidences compared with 2013-2015. In 2016, FoodNet identified 24,029 infections, 5,512 hospitalizations, and 98 deaths caused by these pathogens. The use of culture-independent diagnostic tests (CIDTs) by clinical laboratories to detect enteric pathogens has been steadily increasing since FoodNet began surveying clinical laboratories in 2010 (1). CIDTs complicate the interpretation of FoodNet surveillance data because pathogen detection could be affected by changes in health care provider behaviors or laboratory testing practices (2). Health care providers might be more likely to order CIDTs because these tests are quicker and easier to use than traditional culture methods, a circumstance that could increase pathogen detection (3). Similarly, pathogen detection could also be increasing as clinical laboratories adopt DNA-based syndromic panels, which include pathogens not often included in routine stool culture $(4,5)$. In addition, CIDTs do not yield isolates, which public health officials rely on to distinguish pathogen subtypes, determine antimicrobial resistance, monitor trends, and detect outbreaks. To obtain isolates for infections identified by CIDTs, laboratories must

\footnotetext{
*Connecticut, Georgia, Maryland, Minnesota, New Mexico, Oregon, Tennessee, and selected counties in California, Colorado, and New York (https://www. cdc.gov/foodnet).
}

perform reflex culture ${ }^{\dagger}$; if clinical laboratories do not, the burden of culturing falls to state public health laboratories, which might not be able to absorb that burden as the adoption of these tests increases (2). Strategies are needed to preserve access to bacterial isolates for further characterization and to determine the effect of changing trends in testing practices on surveillance.

FoodNet is a collaboration among CDC, 10 state health departments, the U.S. Department of Agriculture's Food Safety and Inspection Service, and the Food and Drug Administration. FoodNet personnel conduct active, population-based surveillance for laboratory-diagnosed infections

\footnotetext{
${ }_{\dagger}$ Culturing of a specimen with a positive culture-independent diagnostic test (CIDT) result.
}
INSIDE
404 Restaurant Food Allergy Practices - Six Selected Sites, United States, 2014
408 Notes from the Field: Powassan Virus Disease in an Infant - Connecticut, 2016
410 Notes from the Field: Varicella Fatality on a Cargo Vessel — Puerto Rico, 2015
411 Announcements
413 QuickStats

Continuing Education examination available at https://www.cdc.gov/mmwr/cme/conted_info.html\#weekly.

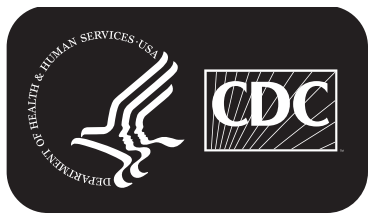


caused by Campylobacter, Cryptosporidium, Cyclospora, Listeria, Salmonella, Shiga toxin-producing Escherichia coli (STEC), Shigella, Vibrio, and Yersinia for 10 sites covering approximately $15 \%$ of the U.S. population (an estimated 49 million persons in 2015). Confirmed bacterial infections are defined as isolation of the bacterium from a clinical specimen by culture. Confirmed parasitic infections are defined as detection of the parasite from a clinical specimen by direct fluorescent antibody test, polymerase chain reaction, enzyme immunoassay, or light microscopy. CIDTs detect bacterial pathogen antigen, nucleic acid sequences, or for STEC, Shiga toxin or Shiga toxin genes, in a stool specimen or enrichment broth. ${ }^{\S}$ A CIDT positiveonly bacterial infection is a positive CIDT result that was not confirmed by culture. Hospitalizations occurring within 7 days of specimen collection are recorded. The patient's vital status at hospital discharge (or 7 days after specimen collection if not hospitalized) is also recorded. Hospitalizations and deaths occurring within 7 days of specimen collection are attributed to the infection. FoodNet also conducts surveillance for physician-diagnosed postdiarrheal hemolytic uremic syndrome (HUS), a potential complication of STEC infection, by review of hospital discharge data through a network of nephrologists and infection preventionists. This report includes HUS cases among persons aged $<18$ years for 2015 , the most recent year with available data.

\footnotetext{
${ }^{\$}$ For Shiga toxin-producing Escherichia coli, only CIDT reports that were positive at a state public health laboratory were counted.
}

Incidence of infection for each pathogen is calculated by dividing the number of infections in 2016 by the U.S. Census estimates of the surveillance area population for 2015 . Incidence is calculated for confirmed infections alone and for confirmed or CIDT positive-only infections combined. A negative binomial model with $95 \%$ confidence intervals (CIs) was used to estimate changes in incidence of confirmed bacterial and parasitic infections and confirmed or CIDT positiveonly bacterial infections in 2016 compared with 2013-2015, adjusting for changes in the surveillance population over time. For STEC, incidence is reported for all STEC serogroups combined because it is not possible to distinguish between serogroups using CIDTs. Insufficient data were available to assess change for Cyclospora. For HUS, the 2015 incidence was compared with incidence during 2012-2014.

\section{Cases of Infection, Incidence, and Trends}

During 2016, FoodNet identified 24,029 cases, 5,512 hospitalizations, and 98 deaths caused by confirmed or CIDT positive-only infections (Table 1). The largest number of confirmed or CIDT positive-only infections in 2016 was reported for Campylobacter $(8,547)$, followed by Salmonella $(8,172)$, Shigella (2,913), STEC $(1,845)$, Cryptosporidium $(1,816)$, Yersinia (302), Vibrio (252), Listeria (127), and Cyclospora (55). The proportion of infections that were CIDT positive without

\footnotetext{
5 Excludes Shiga toxin-positive reports from clinical laboratories that were Shiga toxin-negative at a public health laboratory $(\mathrm{n}=568)$
}

The MMWR series of publications is published by the Center for Surveillance, Epidemiology, and Laboratory Services, Centers for Disease Control and Prevention (CDC), U.S. Department of Health and Human Services, Atlanta, GA 30329-4027.

Suggested citation: [Author names; first three, then et al., if more than six.] [Report title]. MMWR Morb Mortal Wkly Rep 2017;66:[inclusive page numbers].

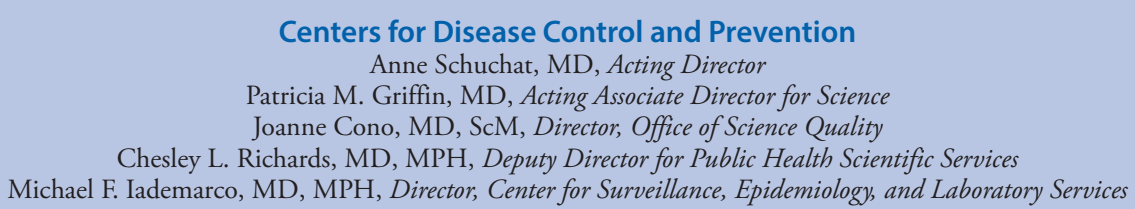

MMWR Editorial and Production Staff (Weekly)

Sonja A. Rasmussen, MD, MS, Editor-in-Chief

Charlotte K. Kent, PhD, MPH, Executive Editor Jacqueline Gindler, MD, Editor

Teresa F. Rutledge, Managing Editor

Douglas W. Weatherwax, Lead Technical Writer-Editor

Stacy A. Benton, Soumya Dunworth, PhD, Teresa M. Hood, MS, Technical Writer-Editors

Timothy F. Jones, MD, Chairman

Matthew L. Boulton, MD, MPH Virginia A. Caine, MD

Katherine Lyon Daniel, $\mathrm{PhD}$

Jonathan E. Fielding, MD, MPH, MBA

David W. Fleming, MD
MMWR Editorial Board

William E. Halperin, MD, DrPH, MPH

King K. Holmes, MD, PhD

Robin Ikeda, MD, MPH

Rima F. Khabbaz, MD

Phyllis Meadows, PhD, MSN, RN

Jewel Mullen, MD, MPH, MPA
Martha F. Boyd, Lead Visual Information Specialist

Maureen A. Leahy, Julia C. Martinroe, Stephen R. Spriggs, Tong Yang, Visual Information Specialists

Quang M. Doan, MBA, Phyllis H. King,

Terraye M. Starr, Moua Yang,

Information Technology Specialists

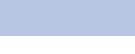


Morbidity and Mortality Weekly Report

TABLE 1. Number of confirmed and CIDT positive-only* bacterial and confirmed parasitic infections, hospitalizations, and deaths, by pathogen FoodNet, 10 U.S. sites, ${ }^{\dagger} 2016^{\S}$

\begin{tabular}{|c|c|c|c|c|c|c|}
\hline \multirow[b]{3}{*}{ Pathogen } & \multicolumn{3}{|c|}{ Confirmed } & \multicolumn{3}{|c|}{ Confirmed or CIDT positive-only } \\
\hline & \multirow[b]{2}{*}{ No. cases } & \multirow{2}{*}{$\begin{array}{c}\text { Hospitalizations } \\
\text { No. }(\%)\end{array}$} & \multirow{2}{*}{$\begin{array}{l}\text { Deaths } \\
\text { No. }(\%)\end{array}$} & \multirow[b]{2}{*}{ No. cases } & \multirow{2}{*}{$\begin{array}{c}\text { Hospitalizations } \\
\text { No. }(\%)\end{array}$} & \multirow{2}{*}{$\frac{\text { Deaths }}{\text { No. (\%) }}$} \\
\hline & & & & & & \\
\hline \multicolumn{7}{|l|}{ Bacteria } \\
\hline Campylobacter & 5,782 & $1,082(19)$ & $10(0.2)$ & 8,547 & $1,697(20)$ & $26(0.3)$ \\
\hline Listeria $a_{1, * * *}$ & 127 & $123(97)$ & $17(13.4)$ & 127 & $123(97)$ & $17(13.4)$ \\
\hline Salmonella & 7,554 & $2,163(29)$ & $39(0.5)$ & 8,172 & $2,255(28)$ & $40(0.5)$ \\
\hline Shigella & 2,256 & $519(23)$ & $2(0.1)$ & 2,913 & $579(20)$ & $2(0.1)$ \\
\hline STEC ${ }^{+\dagger}$ & 1,399 & $326(23)$ & $3(0.2)$ & 1,845 & $408(22)$ & $3(0.2)$ \\
\hline Vibrio & 218 & $61(28)$ & $4(1.8)$ & 252 & $73(29)$ & $4(1.6)$ \\
\hline Yersinia & 205 & $54(27)$ & $3(1.5)$ & 302 & $83(28)$ & $3(1.0)$ \\
\hline \multicolumn{7}{|l|}{ Parasite } \\
\hline Cryptosporidium ${ }^{* *}$ & 1,816 & $291(16)$ & $3(0.2)$ & 1,816 & $291(16)$ & $3(0.2)$ \\
\hline Cyclospora** & 55 & $3(5)$ & $0(-)$ & 55 & $3(5)$ & $0(-)$ \\
\hline Total & 19,412 & 4,622 & 81 & 24,029 & 5,512 & 98 \\
\hline
\end{tabular}

Abbreviations: CIDT = culture-independent diagnostic test; FoodNet = CDC's Foodborne Diseases Active Surveillance Network; STEC $=$ Shiga toxin-producing Escherichia coli.

* CIDT positive-only is defined as detection of the bacterial pathogen, or for STEC, Shiga toxin, or the genes that encode a Shiga toxin, in a stool specimen or enrichment broth using a CIDT. Any positive CIDT result that was confirmed by culture is counted only among the confirmed infections. For STEC, only CIDT reports that were positive at a state public health laboratory were counted.

† Connecticut, Georgia, Maryland, Minnesota, New Mexico, Oregon, Tennessee, and selected counties in California, Colorado, and New York.

$\S$ Data for 2016 are preliminary.

" Listeria cases are defined as isolation of $L$. monocytogenes from a normally sterile site or, in the setting of miscarriage or stillbirth, isolation of $L$. monocytogenes from placental or fetal tissue.

** All Listeria, Cryptosporidium, and Cyclospora infections were confirmed, so confirmed numbers are displayed in both columns.

${ }^{+\dagger}$ For STEC, all serogroups were combined as it is not possible to distinguish between serogroups using CIDTs. Shiga toxin-positive reports from clinical laboratories that were Shiga toxin-negative at a state public health laboratory were excluded $(n=568)$.

culture confirmation in 2016 was largest for Campylobacter (32\%) and Yersinia (32\%), followed by STEC (24\%), Shigella (23\%), Vibrio (13\%), and Salmonella (8\%). The overall increase in CIDT positive-only infections for these six pathogens in 2016 was $114 \%$ (range $=85 \%-1,432 \%$ ) compared with 2013-2015. Among infections with a positive CIDT result in 2016, a reflex culture was attempted on approximately $60 \%$ at either a clinical or state public health laboratory. The proportion of attempted reflex cultures differed by pathogen, ranging from $45 \%$ for Campylobacter to $86 \%$ for STEC and $88 \%$ for Vibrio (Figure). Among infections for which reflex culture was performed, the proportion of infections that were positive was highest for Salmonella (88\%) and STEC (87\%), followed by Shigella (64\%), Yersinia (59\%), Campylobacter (52\%), and Vibrio (46\%).

The incidence of confirmed infections and of confirmed or CIDT positive-only infections per 100,000 persons was highest for Campylobacter (confirmed $=11.79$; confirmed or CIDT positive-only $=17.43)$ and Salmonella $(15.40 ; 16.66)$, followed by Shigella $(4.60 ; 5.94)$, Cryptosporidium (3.64; N/A**), STEC $(2.85 ; 3.76)$, Yersinia $(0.42 ; 0.62)$, and lowest for Vibrio (0.45; 0.51), Listeria (0.26; N/A), and Cyclospora (0.11; N/A) (Table 2). Compared with 2013-2015, the 2016 incidence of Campylobacter infection was significantly lower $(11 \%$ decrease) when including only confirmed infections, yet was

\footnotetext{
** Not applicable: all infections were confirmed.
}

not significantly different when including confirmed or CIDT positive-only infections. Incidence of STEC infection was significantly higher for confirmed infections ( $21 \%$ increase) and confirmed or CIDT positive-only infections ( $43 \%$ increase). Similarly, the incidence of Yersinia infection was significantly higher for both confirmed (29\% increase) and confirmed or CIDT positive-only infections ( $91 \%$ increase). Incidence of confirmed Cryptosporidium infection was also significantly higher in 2016 compared with 2013-2015 (45\% increase).

Among 7,554 confirmed Salmonella cases in 2016, serotype information was available for $6,583(87 \%)$. The most common serotypes were Enteritidis $(1,320 ; 17 \%)$, Newport (797; 11\%), and Typhimurium (704; 9\%). The incidence in 2016 compared with 2013-2015 was significantly lower for Typhimurium (18\% decrease; $\mathrm{CI}=7 \%-21 \%)$ and unchanged for Enteritidis and Newport. Among 208 (95\%) speciated Vibrio isolates, 103 (50\%) were V.parahaemolyticus, 35 (17\%) were $V$. alginolyticus, and 26 (13\%) were $V$. vulnificus. Among 1,394 confirmed and serogrouped STEC cases, 503 (36\%) were STEC O157 and 891 (64\%) were STEC non-O157. Among 586 (70\%) STEC non-O157 isolates, the most common serogroups were O26 (190; 21\%), O103 (178; 20\%), and O111 (106; 12\%). Compared with 2013-2015, the incidence of STEC non-O157 infections in 2016 was significantly higher (26\% increase; CI $=9 \%-46 \%)$ and the incidence of STEC O157 was unchanged. 
FIGURE. Number of infections with positive culture-independent diagnostic test (CIDT) results, ${ }^{*}$ by pathogen, year, and culture status FoodNet, 10 U.S. sites, ${ }^{\dagger}$ 2013-2016 $\$$
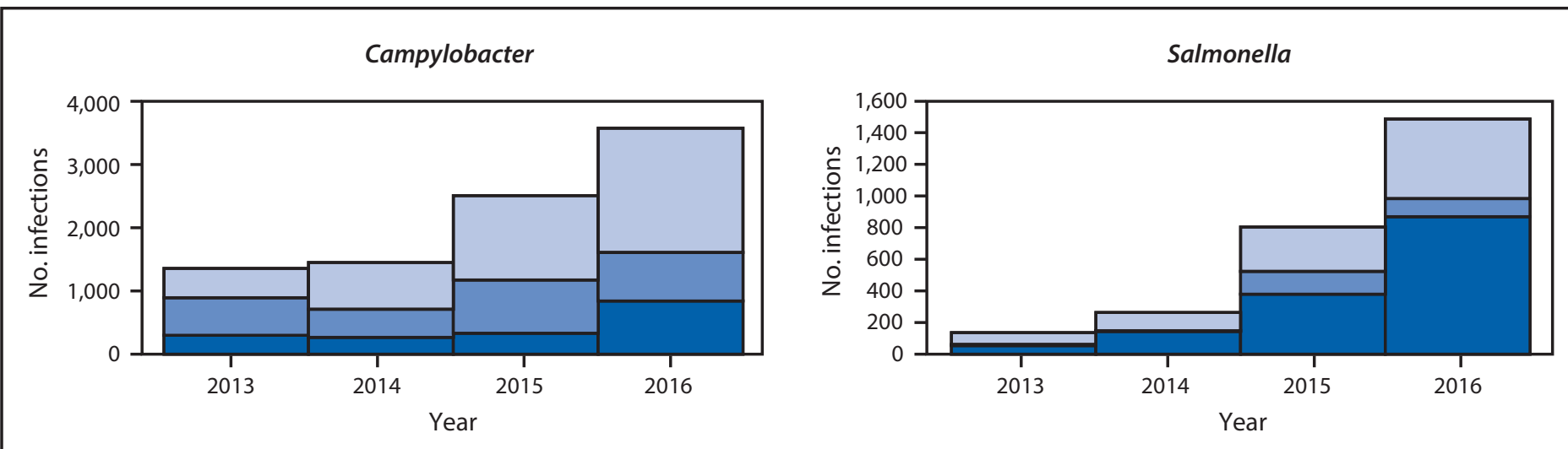

Shigella
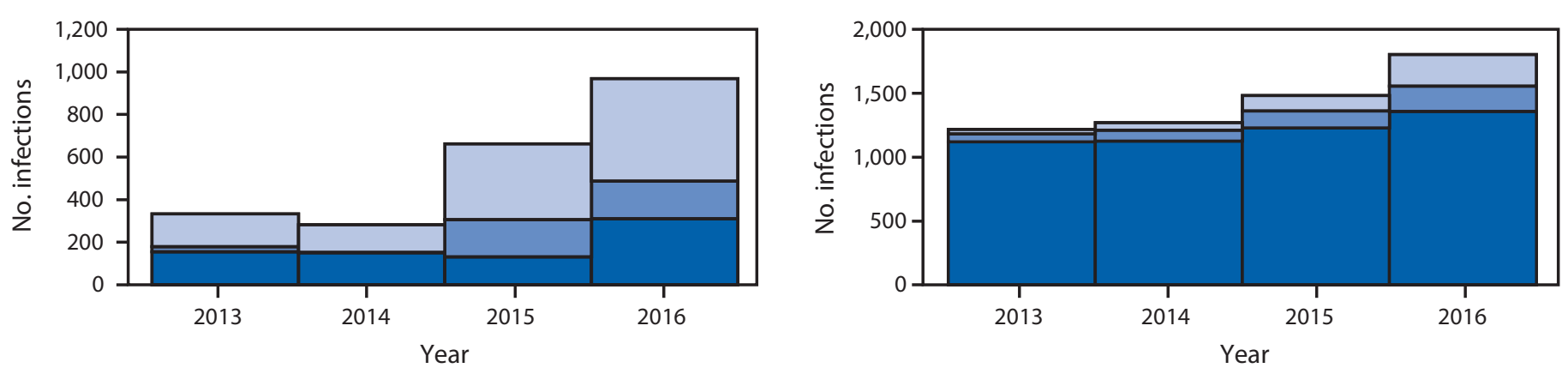

Vibrio
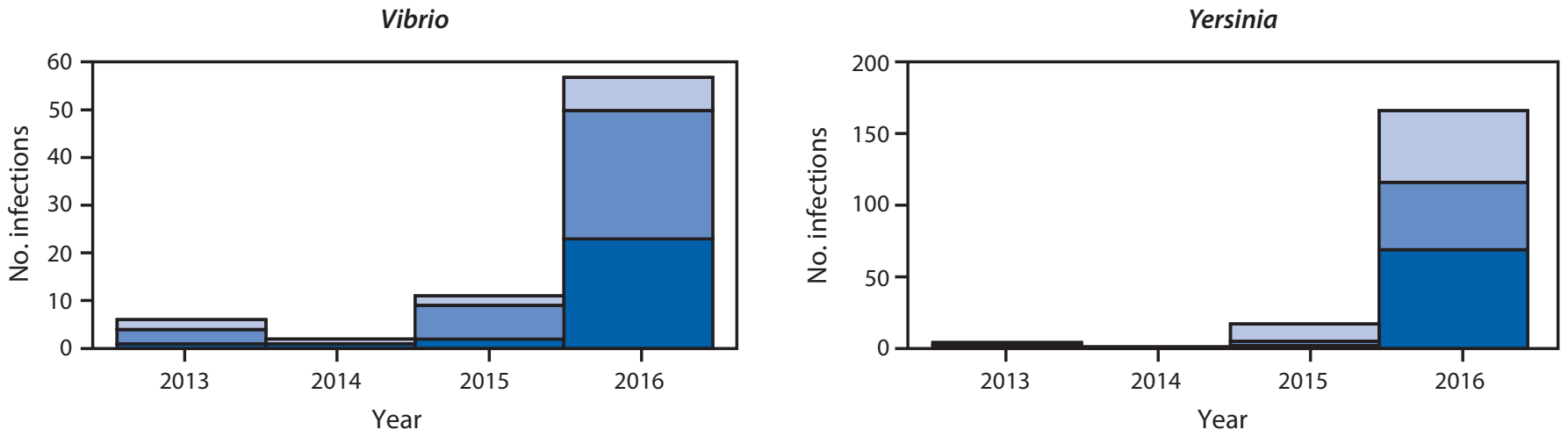

Reflex culture positive

Reflex culture negative

Reflex culture not performed

Abbreviations: FoodNet = CDC's Foodborne Diseases Active Surveillance Network; STEC = Shiga toxin-producing Escherichia coli.

* Positive CIDT results are defined as detection of the bacterial pathogen, or for STEC, Shiga toxin or the genes that encode a Shiga toxin in a stool specimen or enrichment broth using a CIDT. For STEC, only CIDT results that were positive at a state public health laboratory were counted.

† Connecticut, Georgia, Maryland, Minnesota, New Mexico, Oregon, Tennessee, and selected counties in California, Colorado, and New York.

$\S$ Data for 2016 are preliminary.

I" For STEC, all serogroups were combined because distinguishing between serogroups using CIDTs is not possible. Shiga toxin-positive reports from clinical laboratories that were Shiga toxin-negative at a state public health laboratory were excluded $(n=568)$. 
Morbidity and Mortality Weekly Report

TABLE 2. Percentage change in incidence of confirmed and CIDT positive-only* bacterial and confirmed parasitic infections in $2016^{\dagger}$ compared with 2013-2015 average annual incidence, by pathogen — FoodNet, 10 U.S. sites, \$ 2013-2016

\begin{tabular}{|c|c|c|c|c|c|c|}
\hline \multirow[b]{2}{*}{ Pathogen } & \multicolumn{3}{|c|}{ Confirmed } & \multicolumn{3}{|c|}{ Confirmed or CIDT positive-only } \\
\hline & $2016 I^{q}$ & $\%$ Change** & $95 \% \mathrm{Cl}$ & $2016 I^{q}$ & $\%$ Change $^{* *}$ & $95 \% \mathrm{Cl}$ \\
\hline Campylobacter & 11.79 & -11 & -18 to -3 & 17.43 & +3 & -4 to +10 \\
\hline Listeriat† $^{\dagger+}$ & 0.26 & +4 & -18 to +30 & —§§ & —§§ & _ $\S \S$ \\
\hline Salmonella & 15.40 & +2 & -4 to +8 & 16.66 & +6 & -1 to +12 \\
\hline Shigella & 4.60 & +7 & -17 to +38 & 5.94 & +25 & -3 to +62 \\
\hline STEC & 2.84 & +21 & +3 to +42 & 3.76 & +43 & +22 to +67 \\
\hline Vibrio & 0.45 & +2 & -18 to +26 & 0.51 & +16 & -6 to +42 \\
\hline Yersinia & 0.42 & +29 & +2 to +64 & 0.62 & +91 & +52 to +140 \\
\hline Cryptosporidium & 3.70 & +45 & +11 to +89 & —§§ & —§§ & — $\S \S$ \\
\hline
\end{tabular}

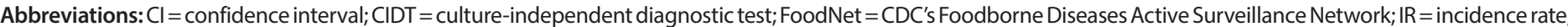
STEC = Shiga toxin-producing Escherichia coli.

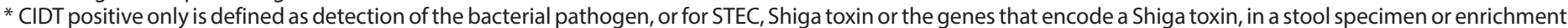

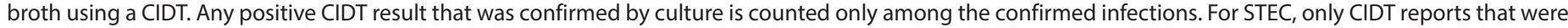
positive at a state public health laboratory were counted.

† Data for 2016 are preliminary.

$\S$ Connecticut, Georgia, Maryland, Minnesota, New Mexico, Oregon, Tennessee, and selected counties in California, Colorado, and New York.

I Per 100,000 population.

** Percentage change reported as increase (+) or decrease (-).

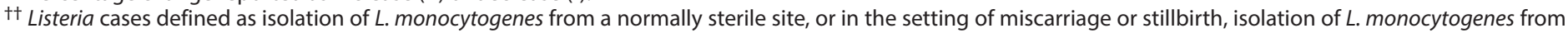
placental or fetal tissue.

$\S \S$ All infections were confirmed.

กๆ For STEC, all serogroups were combined, because it is not possible to distinguish between serogroups using CIDTs.

FoodNet identified 62 cases of postdiarrheal HUS in children aged $<18$ years $(0.56$ cases per 100,000$)$ in 2015 ; $33(56 \%)$ occurred in children aged $<5$ years $(1.18$ cases per $100,000)$. Compared with 2012-2014, in 2015, no significant differences in incidence among all children or children aged $<5$ years were observed.

\section{Discussion}

The number of CIDT positive-only infections reported to FoodNet has been increasing markedly since 2013, as more clinical laboratories adopt CIDTs. Initially, increases were primarily limited to Campylobacter and STEC; followed by substantial increases in Salmonella and Shigella beginning in 2015 (6). The pattern continued in 2016, with large increases in the number of CIDT positive-only Vibrio and Yersinia infections. When including both confirmed and CIDT positive-only infections, incidence rates in 2016 were higher for each of these six pathogens. The increasing use of CIDTs presents challenges when interpreting the corresponding increases in incidence. For example, the incidence of confirmed Campylobacter infections in 2016 was significantly lower than the 2013-2015 average. However, when including CIDT positive-only infections, a slight but not significant increase occurred. For STEC and Yersinia, the incidence of confirmed infections alone and confirmed or CIDT positive-only infections in 2016 were both significantly higher than the 2013-2015 average; the magnitude of change approximately doubled when analyzing CIDT positive-only infections.

Because of the ease and increasing availability of CIDTs, testing for some pathogens might be increasing as health care provider behaviors and laboratory practices evolve (2). Among clinical laboratories in the FoodNet catchment, the use of CIDTs to detect Salmonella, for which the only CIDTs available are DNA-based gastrointestinal syndrome panels, increased from 2 per 460 laboratories $(<1 \%)$ in 2013 to 59 per 421 laboratories (14\%) in 2016 (FoodNet, unpublished data). This increased use paralleled significant increases in incidence of Cryptosporidium, STEC, and Yersinia, and slight but not significant increases in incidence of Campylobacter, Salmonella, Shigella, and Vibrio, all of which are also included in these panel tests. The increase in STEC incidence is driven by the increase in STEC non-O157, which is not typically included in routine stool culture testing because it requires specialized methods. Routine stool cultures performed in clinical laboratories typically include methods that identify only Salmonella, Campylobacter, Shigella, and for some laboratories, STEC O157 (4,5). The increased use of the syndrome panel tests might increase identification, and thus, improve incidence estimates of pathogens for which testing was previously limited.

Results are more quickly obtained using CIDTs than traditional culture methods (3). Because of this, health care providers might be more likely to order a CIDT than traditional culture (2). Increased testing might identify infections that previously would have remained undiagnosed. However, sensitivity and specificity vary by test type. Evaluations of DNA-based syndrome panel tests have indicated high sensitivity and specificity for most targets (3). However, among pathogens for which antigen-based CIDTs are often used, such as Campylobacter and Cryptosporidium, sensitivity and specificity have varied more widely, with a large number of 
false positive results $(7,8)$. Including CIDT positive infections to calculate incidence, some of which could be false positives, might provide an inaccurate estimate. When interpreting incidence and trends in light of changing diagnostic testing, considering frequency of testing, sensitivity, and specificity of these tests is important. The observed increases in incidence of confirmed or CIDT positive-only infections in 2016 compared with 2013-2015 could be caused by increased testing, varying test sensitivity, an actual increase in infections, or a combination of these reasons.

These changes in testing are also important to consider when monitoring progress toward Healthy People 2020 objectives. ${ }^{\dagger \dagger}$ The current objectives were created before the use of CIDTs and were based on confirmed infections. In the future, just as incidence measures should adjust for these changes, objectives should also be evaluated in light of changing diagnostics.

CIDTs pose additional challenges because they do not yield the bacterial isolates necessary for essential public health surveillance activities, such as monitoring trends in pathogen subtypes, conducting molecular testing, detecting outbreaks and implicating vehicles, and determining antimicrobial susceptibility. Reflex culture performed to yield an isolate places an additional burden on laboratories' budgets, personnel, and time. Specimen submission requirements differ by state and pathogen, and this responsibility often falls to state public health laboratories (9). As CIDT use increases and more pathogens are affected, state public health laboratories will be challenged to sufficiently increase their testing capacity and will likely have to prioritize specimens on which to perform reflex culture (10). Clinical laboratories should review state specimen submission requirements and the Association of Public Health Laboratories guidelines ${ }^{\$ \mathbb{S}}$ for reflex culture and submission of CIDT positive specimens.

The findings in this report are subject to at least two limitations. First, the changing diagnostic landscape with unknown changes in frequency of testing, varying test performance, and decreasing availability of isolates for subtyping make interpreting incidence and trends more difficult. Second, changes in health care-seeking behavior, access to health services, or other population characteristics might have changed since the comparison period, which could affect incidence.

Foodborne illness remains a substantial public health concern in the United States. Previous analyses have indicated that the

\footnotetext{
$\dagger_{\dagger}$ https://www.healthypeople.gov/2020/topics-objectives/topic/food-safety/ objectives.

$\$ \$$ https://www.aphl.org/aboutAPHL/publications/Documents/FS-Enteric_ Pathogens_Guidelines_0216.pdf.
}

\section{Summary}

What is already known about this topic?

The incidence of infections transmitted commonly through food has remained largely unchanged for many years. Cultureindependent diagnostic tests (CIDTs) are increasingly used by clinical laboratories to detect enteric infections.

What is added by this report?

Compared with the 2013-2015 average annual incidence, the 2016 incidence of confirmed Campylobacter infections was lower, incidences of confirmed Shiga toxin-producing Escherichia coli (STEC), Yersinia, and Cryptosporidium infections were higher, and incidences of confirmed or CIDT positive-only STEC and Yersinia infections were higher. However, CIDTs complicate the interpretation of surveillance data; testing for pathogens might occur more frequently because of changes in either health care provider behaviors or laboratory testing practices. A large proportion of CIDT positive specimens were not reflex cultured, which is necessary to obtain isolates for distinguishing pathogen subtypes, determining antimicrobial resistance, monitoring trends, and detecting outbreaks.

What are the implications for public health practice?

Some information about the bacteria causing infections, such as subtype and antimicrobial susceptibility, can only be obtained for CIDT positive specimens if reflex culture is performed. Increasing use of CIDTs affects the interpretation of public health surveillance data and ability to monitor progress toward prevention measures.

number of infections far exceeds those diagnosed; CIDTs might be making those infections more visible (11). Most foodborne infections can be prevented, and substantial progress has been made in the past in decreasing contamination of some foods and reducing illness caused by some pathogens. More prevention measures are needed. Surveillance data can provide information on where to target these measures. However, to accurately interpret FoodNet surveillance data in light of changes in diagnostic testing, more data and analytic tools are needed to adjust for changes in testing practices and differences in test characteristics. FoodNet is collecting more data and developing those tools. With these, FoodNet will continue to track the needed progress toward reducing foodborne illness.

\section{Acknowledgments}

Foodborne Diseases Active Surveillance Network staff members, Emerging Infections Program; Brittany Behm, Staci Dixon, Elizabeth Greene, Jennifer Huang, Clare Wise, and FoodNet Fast Development Team, Division of Foodborne, Waterborne, and Environmental Diseases, National Center for Emerging and Zoonotic Infectious Diseases, CDC. 
${ }^{1}$ Division of Foodborne, Waterborne, and Environmental Diseases, National Center for Emerging and Zoonotic Infectious Diseases, CDC; ${ }^{2}$ Oregon Health Authority; ${ }^{3}$ Colorado Department of Public Health and Environment; ${ }^{4}$ Tennessee Department of Health; ${ }^{5}$ University of New Mexico; ${ }^{6}$ Connecticut Department of Public Health; ${ }^{7}$ Maryland Department of Health and Mental Hygiene; ${ }^{8}$ Minnesota Department of Health; ${ }^{9}$ Georgia Department of Public Health; ${ }^{10}$ California Department of Public Health; ${ }^{11}$ New York State Department of Health; ${ }^{12}$ Food Safety and Inspection Service, U.S. Department of Agriculture, Atlanta, Georgia; ${ }^{13}$ Center for Food Safety and Applied Nutrition, Food and Drug Administration, Silver Spring, Maryland.

Corresponding author: Ellyn P. Marder, emarder1@cdc.gov, 404-718-4722.

\section{References}

1. Iwamoto M, Huang JY, Cronquist $\mathrm{AB}$, et al. Bacterial enteric infections detected by culture-independent diagnostic tests-FoodNet, United States, 2012-2014. MMWR Morb Mortal Wkly Rep 2015;64:252-7.

2. Langley G, Besser J, Iwamoto M, et al. Effect of culture-independent diagnostic tests on future Emerging Infections Program surveillance. Emerg Infect Dis 2015;21:1582-8. https://doi.org/10.3201/eid2109.150570

3. Khare R, Espy MJ, Cebelinski E, et al. Comparative evaluation of two commercial multiplex panels for detection of gastrointestinal pathogens by use of clinical stool specimens. J Clin Microbiol 2014;52:3667-73. https://doi.org/10.1128/JCM.01637-14

4. M'ikanatha NM, Dettinger LA, Perry A, Rogers P, Reynolds SM, Nachamkin I. Culturing stool specimens for Campylobacter spp., Pennsylvania, USA. Emerg Infect Dis 2012;18:484-7. https://doi. org/10.3201/eid1803.111266
5. Voetsch AC, Angulo FJ, Rabatsky-Ehr T, et al.; Emerging Infections Program FoodNet Working Group. Laboratory practices for stoolspecimen culture for bacterial pathogens, including Escherichia coli O157:H7, in the FoodNet sites, 1995-2000. Clin Infect Dis 2004;38(Suppl 3):S190-7. https://doi.org/10.1086/381586

6. Huang JY, Henao OL, Griffin PM, et al. Infection with pathogens transmitted commonly through food and the effect of increasing use of culture-independent diagnostic tests on surveillance-Foodborne Diseases Active Surveillance Network, 10 U.S. sites, 2012-2015. MMWR Morb Mortal Wkly Rep 2016;65:368-71. https://doi.org/10.15585/mmwr.mm6514a2

7. Fitzgerald C, Patrick M, Gonzalez A, et al. Multicenter evaluation of clinical diagnostic methods for detection and isolation of Campylobacter spp. from stool. J Clin Microbiol 2016;54:1209-15. https://doi. org/10.1128/JCM.01925-15

8. Roellig DM, Yoder JS, Madison-Antenucci S, et al. Community laboratory testing for Cryptosporidium: multicenter study retesting public health surveillance stool samples positive for Cryptosporidium by rapid cartridge assay with direct fluorescent antibody testing. PLoS One 2017;12:e0169915. https://doi.org/10.1371/journal.pone.0169915

9. Association of Public Health Laboratories. State legal requirements for submission of isolates and other clinical materials by clinical laboratories: a review of state approaches. Silver Spring, MD: Association of Public Health Laboratories; 2015. https://www.aphl.org/aboutAPHL/ publications/Documents/StateRequirements_Appendix_v6.pdf

10. Shea S, Kubota KA, Maguire H, et al. Clinical microbiology laboratories' adoption of culture-independent diagnostic tests is a threat to foodbornedisease surveillance in the United States. J Clin Microbiol 2016;55:10-9. https://doi.org/10.1128/JCM.01624-16

11. Scallan E, Hoekstra RM, Angulo FJ, et al. Foodborne illness acquired in the United States - major pathogens. Emerg Infect Dis 2011;17:7-15. https://doi.org/10.3201/eid1701.P11101 